\title{
The Evaluation of Efficacy and Side Effects of Radiotherapy in Cerebral Meningiomas
}

\author{
Timur Koca*, Rahmi Atil Aksoy, Yasemin Sengün, Melek Gamze Aksu, Nina Tuncel, \\ Mine Genc Ozay, Aylin Fidan Korcum
}

Department of Radiation Oncology, School of Medicine, Akdeniz University, Konyaalt1, Turkey

Email: ^timurkoca3@gmail.com

How to cite this paper: Koca, T., Aksoy, R.A., Sengün, Y., Aksu, M.G., Tuncel, N., Ozay, M.G. and Korcum, A.F. (2020) The Evaluation of Efficacy and Side Effects of Radiotherapy in Cerebral Meningiomas. International Journal of Medical Physics, Clinical Engineering and Radiation Oncology, 9, 157-164.

https://doi.org/10.4236/ijmpcero.2020.94014

Received: August 13, 2020

Accepted: September 25, 2020

Published: September 28, 2020

Copyright $\odot 2020$ by author(s) and Scientific Research Publishing Inc. This work is licensed under the Creative Commons Attribution International License (CC BY 4.0).

http://creativecommons.org/licenses/by/4.0/

\begin{abstract}
Aims: The current study aimed to evaluate the efficacy, side effects of radiotherapy and factors influencing treatment outcome in patients received radiotherapy for cerebral meningiomas. Methods and Material: In the last two decades, a total of 35 patients with cerebral meningioma who received radiotherapy in our clinic were evaluated statistically in terms of survival, toxicity and prognostic factors. The records of patients diagnosed with cerebral meningioma who underwent postoperative radiotherapy were retrospectively analyzed. Statistical analysis used: Statistical comparisons were made using IBM SPSS v24.0. Kaplan-Meier method was used to compare survival times. Results: Five of the patients had grade I meningioma showing recurrence, 18 were grade II meningioma and 12 were grade III meningioma. Patients were treated with 54 or 60 Gy adjuvant radiotherapy with 2 Gy daily fractions according to histopathological grade and operation type. Three and five-year overall survival rates were $56 \%$ and $40 \%$, respectively and median overall survival was 36 months. Progression was observed in 15 of the 35 patients included in the study. Three and five-year progression-free survival (PFS) rates of the patients were $56 \%$ and $50 \%$, respectively. There was a statistically significant correlation between histopathological grade and overall survival among patients. However; no statistical difference found in overall survival of patients in terms of tumor location, operation type and age. Conclusions: In the treatment of cerebral meningiomas, changes in radiotherapy dose and field designs can be predicted according to the tumor grade and operation type. In addition, it is thought that large scale studies are needed to determine prognostic factors more meticulously.
\end{abstract}

\section{Keywords}

Brain Tumor, Cerebral Meningioma, Atypical Meningioma, Anaplastic Meningioma, Radiotherapy, Overall Survival 


\section{Introduction}

Meningiomas are the most common central nervous system tumors in adults, constituting $36.8 \%$ of central nervous system located tumors and are more common in women [1]. Histopathological grade, tumor size, tumor localization and age have been demonstrated as prognostic factors in cerebral meningiomas [2].

Meningiomas are divided into three pathological grades according to the World Health Organization (WHO) definition [3] [4]. 80\% of cerebral meningiomas are grade I, $20 \%$ are grade II and III [1]. Recurrence and progression are observed earlier in high grade meningiomas than others [5]. Resection size in cerebral meningioma is classified using the Simpson grading system, Simpson grade I - III is evaluated as gross total resection and Simpson grade IV - V subtotal resection [6].

Although the removal of tumor is accepted as the primary treatment, the necessity of postoperative radiotherapy after tumor resection depends on the presence of residual tumor and tumor grade [7]. While the use of radiotherapy in benign meningiomas is controversial, postoperative radiotherapy is recommended in anaplastic and clinically aggressive atypical meningiomas [8] [9]. Radiotherapy is preferred as the primary treatment in patients with severe comorbid conditions and in patients where tumor localization is risky for surgery [10].

In our study, prognostic factors, survival results and radiotherapy-related side effects were evaluated in patients with cerebral meningioma who underwent postoperative radiotherapy.

\section{Method}

A total of 35 patients, 22 women and 13 men, who received postoperative radiotherapy due to cerebral meningioma between 2000 and 2019, were included in this study. The age range of patients varies between 20 and 77 (median: 55). Patients aged 20 years and above who were operated for cerebral meningioma and received postoperative radiotherapy were included in the study. Patients with extracranial meningioma, multiple meningioma, optic nerve meningioma, previously receiving cranial radiotherapy, patients with neurofibromatosis, patients with severe comorbid disease and patients under 20 years of age were not included in the study. Patient examination data and hospital medical records were used to obtain clinical evaluation and radiotherapy data, side effect evaluations and survival results after radiotherapy.

In the pre-treatment patient simulations, customized thermoplastic head and neck masks were used to provide immobilization, and computerized tomography sections were taken from the patients in a supine position, $2 \mathrm{~mm}$ section thickness. Nineteen patients who received treatment before 2009 received 2-dimensional conformal radiotherapy with linear accelerator device and 16 patients who received treatment in 2009 and after, 3-dimensional conformal radiotherapy or Intensity Modulated Radiation Therapy (IMRT) were performed. Patients with recurrent postoperative grade I meningioma, subtotal or gross total operative 
grade II and III meningioma cases were included in the study and 54 or 60 Gy adjuvant radiotherapy was applied according to histopathological grade and operation type.

During radiotherapeutical planning, brainstem, spinal cord, eyes, lenses, optic nerves, optic chiasm, cochleae were defined as organs at risk. To achieve the planned risk volume (PRV), $0.3 \mathrm{~cm}$ margin was given to the organ volume. Gross tumor volume (GTV) of the patients were created by cross-section drawing in contouring tomography via pre-op and post-op magnetic resonance images (MRI). Cerebral edema is not included in GTV. Clinical target volume (CTV) was created by giving $1-2 \mathrm{~cm}$ margin to tumor bed and planning target volume (PTV) was created by giving $3-5 \mathrm{~mm}$ margin to CTV. During the planning, when critical levels of organs at risk volumes were exceeded, manual corrections of PTV volume up to $3 \mathrm{~mm}$ were made.

All patients were systemically examined once a week for acute reactions during the radiotherapy process. After radiotherapy, patients were called to routine controls in the first month, and every three months in the first two years, and the brain was followed up with MRI. After the second year, patients were evaluated every six months for five years, by examination and brain MRI. Treatment responses were evaluated according to neuro-oncological criteria. Early and late side effects were evaluated according to Common Terminology Criteria for Adverse Events v3.0 grading system.

Statistical comparisons were made using IBM SPSS v24.0. Descriptive analyzes were used to classify patients. The overall survival evaluation was based on the duration from the operation date to the date of death for all patients. PFS was calculated based on the time from the operation date to the date of progression. Kaplan-Meier method was used to compare survival times. Possible prognostic factors such as age, histopathological grade, tumor resection pattern, tumor location were also analyzed. Log-rank test was used to identify predictors of survival by univariate analysis. Statistically, $\mathrm{p}$ value of 0.05 or less was considered significant. This study was approved by Akdeniz University Faculty of Medicine Clinical Research Ethics Committee on 27.11.2019 (Decision No. 1097).

\section{Results}

Eighteen of the patients included in the study had frontal, 11 were parietooccipital and 6 were temporally located meningioma. Gross total excision performed in 11 patients and subtotal excision for remaining 24 patients. Five of the patients were grade I recurrent meningioma, 18 were grade II and 12 were grade III meningioma. Grade I and grade II meningiomas are included in the low grade group, grade III meningiomas are included in the high grade group.

Overall survival rates of one, two, three and five years were $90 \%, 74 \%, 56 \%$, $40 \%$, respectively and median overall survival was 36 months. While the median overall survival was 93 months in the low grade meningioma group and 21 months in the high grade meningioma group. Overall survival was statistically 
significantly better in the low grade group than high grade group (Table 1).

When gross total excision and subtotal excision groups were compared statistically, no significant difference was found between the two groups in terms of overall survival. When the relationship between tumor location and overall survival is evaluated, median overall survivals were; 36 months in the frontally located meningiomas, 58 months in the parietooccipital localization and was 93 months in the temporally located group. Statistically, no significant difference was found between the 3 groups. Median overall survival was 58 months under the age of 55, 26 months at the age of 55 and above, but when the two groups were compared statistically, there was no significant difference between them (Table 1).

Progression was observed in 15 of the 35 patients included in the study. One, two, three and five-year PFS of the patients were $84 \%, 60 \%, 56 \%$ and $50 \%$, respectively. A total of 18 patients died, 11 of the low grade meningiomas and 7 of the high grade meningiomas. Fifteen patients died due to disease and 3 patients died non-disease reasons.

The most common acute side effect was skin toxicity, seen in 26 patients. Alopecia was observed in 14 of these patients and dermatitis in 12. Headache was observed in 15 patients and loss of balance in 4 patients. Among the late side effects, cognitive disorders were the most common.

Four patients had seizures in the post radiotherapy period and one patient had decreased vision. Corticosteroids and other necessary medications have been used in the prophylaxis and treatment of cerebral edema. Acute and late side effects of the radiotherapy are summarised in the Table 2.

Table 1. Statistical evaluations of prognostic factors for cerebral menengiomas $\left(^{\star}\right.$ : Number of patients).

\begin{tabular}{|c|c|c|c|c|}
\hline Variable & $\mathrm{n}^{*}$ & $\%$ & Overall Survival (Months) & $\mathrm{p}$ value \\
\hline Histopathological Type & & & & 0.03 \\
\hline Low Grade & 23 & $66 \%$ & 93 & \\
\hline High Grade & 12 & $34 \%$ & 21 & \\
\hline Operation Type & & & & 0.9 \\
\hline Gross total excision & 11 & $31 \%$ & 36 & \\
\hline Subtotal excision & 24 & $69 \%$ & 36 & \\
\hline Tumor Localization & & & & 0.85 \\
\hline Frontal & 18 & $52 \%$ & 36 & \\
\hline Parieto-occipital & 11 & $31 \%$ & 58 & \\
\hline Temporal & 6 & $17 \%$ & 93 & \\
\hline Age & & & & 0.3 \\
\hline$<55$ & 16 & $45 \%$ & 58 & \\
\hline$\geq 55$ & 19 & $55 \%$ & 26 & \\
\hline
\end{tabular}


Table 2. Acute and late side effects of the radiotherapy ( $†$ : Grade).

\begin{tabular}{cccccc}
\hline Acute Side Effects & G 1 & G2 & G3 & G4 & Sum \\
\hline Alopecia & 8 & 6 & 0 & 0 & 14 \\
Dermatitis & 7 & 5 & 0 & 0 & 12 \\
Headache & 10 & 5 & 0 & 0 & 15 \\
Balance Loss & 2 & 1 & 1 & 0 & 4 \\
Late Side Effects & G1 & G2 & G3 & G4 & Sum \\
Cognitive disorder & 12 & 7 & 0 & 0 & 19 \\
Seizure & 1 & 2 & 1 & 0 & 4 \\
Decreased vision & 0 & 0 & 1 & 0 & 1 \\
\hline
\end{tabular}

\section{Discussion}

Gross total tumor excision including dura is recommended as the first-line treatment for cerebral meningiomas [7]. Although there is a consensus that anaplastic meningiomas should receive adjuvant radiotherapy, the role of adjuvant radiotherapy in the treatment of atypical meningiomas has long been the subject of debate [11] [12]. Many studies have been conducted to determine the effects of adjuvant radiotherapy on PFS and overall survival in cerebral meningiomas [13] [14] [15] [16].

Milosevic et al. showed an improvement in overall survival for atypical and anaplastic meningiomas with adjuvant radiotherapy at doses of $50 \mathrm{~Gy}$ and above. Radiotherapy was recommended to all patients in the early postoperative period [13].

Goldsmith et al. suggested that adjuvant radiotherapy should be the standard treatment following subtotal resection in their study with 140 patients with cerebral meningioma. They reported that if postoperative radiotherapy is postponed until recurrence, the effectiveness of the treatment decreases as the tumor undergoes malignant transformation or tumor size increases [14].

In a study of 2515 patients with atypical meningioma, Wang et al. showed that adjuvant radiotherapy after subtotal resection provided a statistically significant increase in overall survival and reported that adjuvant radiotherapy after gross total resection did not significantly improve overall survival [15].

In a study evaluating 45 patients with atypical meningioma who underwent gross total resection, patients were divided into two groups as those who received adjuvant radiotherapy and those who were observed. Thirty two of the patients were in the observation group and 13 were in the adjuvant radiotherapy group. Median PFS was 19 months in the observation group, and 52.5 months in the adjuvant radiotherapy group [16].

In the study of Rogers et al. which included 53 patients (RTOG 0539), patients were divided into three risk groups depending on the histopathological grade, type of operation, and the presence of recurrence. High-risk meningioma group included grade III meningiomas of any resection extent, grade II meningiomas 
with subtotal resection and recurrent grade II meningiomas of any resection extent. Adjuvant radiotherapy from 60 Gy high dose and 54 Gy low dose areas was applied to this high risk group with simultaneous integrated boost (SIB) technique. In the study, 3-year PFS was the primary endpoint, the 3-year PFS rate was $59 \%$, and the 5 -year PFS rate was $47 \%$. In the study, the 3 -year overall survival rate was $78 \%$ and the 5-year overall survival rate was 59\% [17]. In our study, the selected patients were mostly in the subtotal resection group, and the majority of patients who underwent gross total resection were grade III meningiomas. These patients received 54 or 60 Gy adjuvant radiotherapy and 3-year PFS rate was $56 \%$ and 5-year PFS rate was $50 \%$. The 3-year overall survival rate was $56 \%$, the 5 -year overall survival rate was $40 \%$. It was thought that the probable cause of these survival differences might be other factors belonging to the patients (race, diet, etc.) or SIB technique was not used in our study.

Some studies have shown that patients undergoing gross total resection are associated with a longer overall survival than patients with subtotal resection [18] [19]. In the study, which included 119 patients with atypical and anaplastic meningioma Pasquie et al. reported that the extent of surgical resection was not a significant prognostic factor [20]. Similarly, no significant relationship was found between the type of operation and the overall survival time in our cases. However, this may be related to performing statistical analyzes for the extent of surgical resection in the whole group. Subgroup analyzes might reveal, the effect of surgical resection extent considering tumor grade.

In a study conducted with 74 patients (33 atypical, 41 anaplastic meningiomas) evaluating the prognostic effects of clinicopathological features of the atypical and anaplastic meningiomas, the overall survival time was 142 months in the atypical meningioma group and 39 months in the anaplastic meningioma group, and a statistically significant difference was found between the two groups [21]. Similarly, in our study, the overall survival time was found to be significantly longer in the low grade meningioma group than in the high grade meningioma group.

Again in a series of 45 cases in which prognostic factors were evaluated in patients with atypical meningioma, the overall survival time was found to be significantly lower in the patient group over 60 years of age [22]. This situation is similar to our study, and in our study, the overall survival time was lower in the group over 55 years of age. However, this difference was not statistically significant $(\mathrm{p}=0.3)$.

\section{Comments}

In the treatment of cerebral meningiomas, changes in the radiotherapy dose and field designs can be predicted according to tumor grade and type of operation. This study reflects a single center experience which includes regional patient data. The results of this study are expected to contribute data to the radiotherapy of patients with cerebral meningioma. It is important to conduct meta-analysis of 
studies by collecting multiple center data. With future multicentre studies, radiotherapy doses, toxicity and survival data will be rationalized in cerebral meningioma treatments.

\section{Conflicts of Interest}

The authors declare no conflicts of interest regarding the publication of this paper.

\section{References}

[1] Ostrom, Q.T., Gittleman, H., Liao, P., Vecchione-Koval, T., Wolinsky, Y., Kruchko, C. and Barnholtz-Sloan, J.S. (2017) CBTRUS Statistical Report: Primary Brain and Other Central Nervous System Tumors Diagnosed in the United States in 20102014. Neuro-Oncology, 19, v1-v88. https://doi.org/10.1093/neuonc/nox158

[2] Domingues, P.H., Sousa, P., Otero, Á., Gonçalves, J.M., Ruiz, L., De Oliveira, C., Lopes, M.C., Orfao, A. and Tabernero, M.D. (2014) Proposal for a New Risk Stratification Classification for Meningioma Based on Patient Age, WHO Tumor Grade, Size, Localization, and Karyotype. Neuro-Oncology, 16, 735-747. https://doi.org/10.1093/neuonc/not325

[3] Kleihues, P., Burger, P.C. and Scheithauer, B.W. (1993) The New WHO Classification of Brain Tumours. Brain Pathology, 3, 255-268. https://doi.org/10.1111/j.1750-3639.1993.tb00752.x

[4] Louis, D.N., Perry, A., Reifenberger, G., Von Deimling, A., Figarella-Branger, D., Cavenee, W.K., Ohgaki, H., Wiestler, O.D., Kleihues, P. and Ellison, D.W. (2016) The 2016 World Health Organization Classification of Tumors of the Central Nervous System: a summary. Acta Neuropathologica, 131, 803-820. https://doi.org/10.1007/s00401-016-1545-1

[5] Sun, S.Q., Hawasli, A.H., Huang, J., Chicoine, M.R. and Kim, A.H. (2015) An Evidence-Based Treatment Algorithm for the Management of WHO Grade II and III Meningiomas. Neurosurgical Focus, 38, E3. https://doi.org/10.3171/2015.1.FOCUS14757

[6] Simpson, D. (1957) The Recurrence of Intracranial Meningiomas after Surgical Treatment. Journal of Neurology, Neurosurgery and Psychiatry, 20, 22-39.

[7] Goldbrunner, R., Minniti, G., Preusser, M., Jenkinson, M.D., Sallabanda, K., Houdart, E., Von Deimling, A., Stavrinou, P., Lefranc, F., Lund-Johansen, M., Moyal, E.C., Brandsma, D., Henriksson, R., Soffietti, R. and Weller, M. (2016) EANO Guidelines for the Diagnosis and Treatment of Meningiomas. The Lancet Oncology, 17, e383-e391. https://doi.org/10.1016/S1470-2045(16)30321-7

[8] Al-Mefty, O., Kersh, J.E., Routh, A. and Smith, R.R. (1990) The Long-Term Side Effects of radiation Therapy for Benign Brain Tumors in Adults. Journal of Neurosurgery, 73, 502-512. https://doi.org/10.3171/jns.1990.73.4.0502

[9] Coke, C.C., Corn, B.W., Werner-Wasik, M., Xie, Y. and Curran Jr., W.J. (1998) Atypical and Malignant Meningiomas: An Outcome Report of Seventeen Cases. Journal of Neurosurgery, 39, 65-70. https://doi.org/10.1023/A:1005981731739

[10] Ge, Y.L., Liu, D., Zhang, Z.Y., Li, Y.H., Lin, Y.G., Wang, G.K., Zong, Y.Q. and Liu, E.H. (2019) Gamma Knife Radiosurgery for Intracranial Benign Meningiomas: Follow-Up Outcome in 130 Patients. Neurosurgical Focus, 46, E7. https://doi.org/10.3171/2019.3.FOCUS1956

[11] Mesic, J.B., Hanks, G.E. and Doggett, R.L. (1986) The Value of Radiation Therapy 
as an Adjuvant to Surgery in Intracranial Meningiomas. American Journal of Clinical Oncology, 9, 337-340. https://doi.org/10.1097/00000421-198608000-00013

[12] Dziuk, T.W., Woo, S., Butler, E.B., et al. (1998) Malignant Meningioma: An Indication for Initial Aggressive Surgery and Adjuvant Radiotherapy. Journal of Neurosurgery, 37, 177-188. https://doi.org/10.1023/A:1005853720926

[13] Milosevic, M.F., Frost, P.J., Laperriere, N.J., Wong, C.S. and Simpson W.J. (1996) Radiotherapy for Atypical or Malignant Intracranial Meningioma. International Journal of Radiation Oncology, Biology, Physics, 34, 817-822.

https://doi.org/10.1016/0360-3016(95)02166-3

[14] Goldsmith, B.J., Wara, W.M., Wilson, C.B., Larson, D.A. (1994) Postoperative Irradiation for Subtotally Resected Meningiomas. A Retrospective Analysis of $140 \mathrm{~Pa}$ tients Treated from 1967 to 1990. Journal of Neurosurgery, 80, 195-201. https://doi.org/10.3171/jns.1994.80.2.0195

[15] Wang, C., Kaprealian, T.B., Suh, J.H., Kubicky, C.D., Ciporen, J.N., Chen Y. and Jaboin, J.J. (2017) Overall survival Benefit Associated with Adjuvant Radiotherapy in WHO Grade II Meningioma. Neuro-Oncology, 19, 1263-1270. https://doi.org/10.1093/neuonc/nox007

[16] Komotar, R.J., Iorgulescu, J.B., Raper, D.M., Holland, E.C., Beal, K., Bilsky, M.H., Brennan, C.W., Tabar, V., Sherman, J.H., Yamada, Y. and Gutin, P.H. (2012) The Role of Radiotherapy Following Gross-Total Resection of Atypical Meningiomas. Journal of Neurosurgery, 117, 679-686. https://doi.org/10.3171/2012.7.JNS112113

[17] Rogers, C.L., Won, M., Vogelbaum, M.A., Perry, A., Ashby, L.S., Modi, J.M., Alleman, A.M., Galvin, J., Fogh, S.E., Youssef, E., Deb, N., Kwok, Y., Robinson, C.G., Shu, H.K., Fisher, B.J., Panet-Raymond, V., McMillan, W.G., De Groot, J.F., Zhang, P. and Mehta, M.P. (2020) High-Risk Meningioma: Initial Outcomes From NRG Oncology/RTOG 0539. International Journal of Radiation Oncology, Biology, Physics, 106, 790-799. https://doi.org/10.1016/j.ijrobp.2019.11.028

[18] Zaher, A., Abdelbari Mattar, M., Zayed, D.H., Ellatif, R.A. and Ashamallah, S.A. (2013) Atypical Meningioma: A Study of Prognostic Factors. World Neurosurgery, 80, 549-553. https://doi.org/10.1016/j.wneu.2013.07.001

[19] Zhao, P., Hu, M.Q., Zhao, M., Ren, X.H. and Jiang, Z.L. (2015) Prognostic Factors for Patients with Atypical or Malignant Meningiomas Treated at a Single Center. Neurosurgical Review, 38, 101-107. https://doi.org/10.1007/s10143-014-0558-2

[20] Pasquier, D., Bijmolt, S., Veninga, T., Rezvoy, N., Villa, S., Krengli, M., Weber, D.C., Baumert, B.G., Canyilmaz, E., Yalman, D., Szutowicz, E., Tzuk-Shina, T. and Mirimanoff, R.O. (2008) Rare Cancer Network. Atypical and Malignant Meningioma: Outcome and Prognostic Factors in 119 Irradiated Patients. A Multicenter, Retrospective Study of the Rare Cancer Network. International Journal of Radiation Oncology, Biology, Physics, 71, 1388-1393. https://doi.org/10.1016/j.ijrobp.2007.12.020

[21] Yang, S.Y., Park, C.K., Park, S.H., Kim, D.G., Chung, Y.S. and Jung, H.W. (2008) Atypical and Anaplastic Meningiomas: Prognostic Implications of Clinicopathological Features. Journal of Neurology, Neurosurgery and Psychiatry, 79, 574-580. https://doi.org/10.1136/jnnp.2007.121582

[22] Endo, T., Narisawa, A., Ali, H.S., Murakami, K., Watanabe, T., Watanabe, M., Jokura, H., Endo, H., Fujimura, M., Sonoda, Y. and Tominaga, T. (2016) A Study of Prognostic Factors in 45 Cases of Atypical Meningioma. Acta Neurochirurgica, 158, 1661-1667. https://doi.org/10.1007/s00701-016-2900-7 